\title{
The Mini Nutritional Assessment (MNA) predicts care need in older Taiwanese: results of a national cohort study
}

\author{
Alan C. Tsai ${ }^{1,2 *}$, Wei-Chung Hsu ${ }^{1,3}$ and Jiun-Yi Wang ${ }^{1,4}$ \\ ${ }^{1}$ Department of Healthcare Administration, Asia University, 500 Liufeng Road, Wufeng, Taichung 41354, Taiwan, ROC \\ ${ }^{2}$ Department of Health Services, School of Public Health, China Medical University, Taichung, Taiwan, ROC \\ ${ }^{3}$ Department of Radiation Oncology, Chung-Kang Branch, Cheng-Ching General Hospital, Taichung, Taiwan, ROC \\ ${ }^{4}$ Center for Health Policy and Management Research, Asia University, Taichung, Taiwan, ROC \\ (Submitted 30 July 2013 - Final revision received 6 January 2014 - Accepted 7 January 2014 - First published online 10 March 2014)
}

\section{Abstract}

The present study determined the ability of the Mini Nutritional Assessment (MNA) to predict care need in older people. We analysed the datasets of the Taiwan Longitudinal Study on Aging. The 1999 survey containing the MNA items served as the baseline and the 2003 survey served as the endpoint. Of the 4440 participants, 2890 were aged $\geq 65$ years and served as subjects in the present study. After excluding 150 subjects having incomplete data, 2740 were rated for nutritional status with the normalised long-form (LF) and short-form (SF) MNATaiwan version 1 (T1) and version 2 (T2) and evaluated with logistic regression analysis for cross-sectional associations of the rated nutritional status with care need, controlled for age, sex, education level, living arrangement and physical activity. Receiver operating characteristic curves were generated for evaluating the ability of the MNA to predict care need. After further excluding 250 subjects who had care need at baseline and seventy-six who were lost to follow-up, 2414 were evaluated for the ability of the MNA to predict subsequent care need with logistic regression analysis. The results demonstrated that all the MNA predicted concurrent and subsequent care need well. The OR for needing subsequent care in the 'at-risk' and 'malnourished' groups were, respectively, 2.04 and 3.33 for the MNA-T1-LF, 2.10 and 5.35 for the MNA-T2-LF, 1.49 and 2.48 MNA-T1-SF, and 1.80 and 3.44 for the MNA-T2-SF (all $P<0.05$ ), and the respective Nagelkerke $R^{2}$ values were $0 \cdot 190,0 \cdot 191,0 \cdot 184$ and $0 \cdot 192$. In conclusion, all the four MNA have the ability to predict future care need, including the MNA-T2-SF, which appears to have great potential for practical applicability.

\section{Key words: Care need: Older people: Mini Nutritional Assessment: Nutritional status: Taiwan}

The Mini Nutritional Assessment (MNA) was developed for assessing/screening the risk of malnutrition in older people approximately two decades ago ${ }^{(1-3)}$. The full (or long-form, LF) MNA comprises eighteen items grouped into four rubrics: anthropometrics; global indicators; dietary intake status; selfview of health and nutritional status. The MNA also has a short form (SF), which was originally developed to allow the use of a two-step, screening and then assessment, process in low-risk populations. The MNA-SF was derived from the six key items in the full MNA, and its ability to predict undernutrition generally parallels that of the full $\mathrm{MNA}^{(4)}$. Because of its high performance, the MNA-SF has been proposed to serve as a stand-alone unit ${ }^{(5)}$. Recently, we have also shown that calf circumference (CC) can substitute BMI and improve the predictive ability of the scale ${ }^{(5,6)}$. Thus, the MNA has an alternative version, the CC-MNA.

The MNA was developed based on the clinical data of Caucasian populations. Due to anthropometric and cultural differences, we normalised and validated the respective versions for Taiwanese by adopting population-specific anthropometric cut-off points and dietary habits. The MNATaiwan version 1 (MNA-T1) and MNA-Taiwan version 2 (MNA-T2), which are equivalent to the original MNA and CC-MNA, respectively ${ }^{(7,8)}$, are two versions specifically normalised for Taiwanese.

The MNA has multifunctional properties. In addition to rating nutritional risk status, it also rates functional and cognitive status, or frailty, and it can predict subsequent functional and cognitive declines, the risk of falling, hospitalisation outcomes, length of hospital stay, and mortality ${ }^{(9,10)}$. The fact that the MNA is able to predict subsequent functional and cognitive declines is of particular significance.

Populations around the world are ageing rapidly. Older people have an increased risk of malnutrition. Malnutrition can increase functional and cognitive impairments and difficulties in eating. Advanced ageing would almost eventually

Abbreviations: ADL, Activities of Daily Living; CC, calf circumference; MNA, Mini Nutritional Assessment; LF, long form; SF, short form; T1, Taiwan version 1; T2, Taiwan version 2; TLSA, Taiwan Longitudinal Study on Aging.

*Corresponding author: A. C. Tsai, fax +886 42332 1206, email atsai@umich.edu 
lead to frailty, a state of increased vulnerability resulting from multisystemic reduction of physiological capacities. Severe frailty will lead to dependency and increased care need.

We theorise that if the MNA is able to predict malnutrition and subsequent functional impairment, cognitive declines and frailty, then it should be able to predict care need. Thus, the present study was conducted to determine the ability of the MNA to predict care need. As our earlier studies have suggested that the MNA-SF is quite effective at predicting functional decline and the MNA-T2 (CC-MNA) is superior to the original MNA under most conditions ${ }^{(6,11)}$, we also compared the predictive abilities of these versions.

\section{Methods}

\section{Source of data}

In the present study, datasets of the 'Taiwan Longitudinal Study on Aging' (TLSA), a population-representative cohort study, were analysed ${ }^{(12)}$. The TLSA employed a multistage equal probability sampling process based on the national household registration comprising personal data and residential addresses of all citizens. Stage one involved random selection of administrative units (townships) to represent the population; stage two involved proportionate-to-size random selection of lins (groups of neighbouring household units as the basic administrative unit) from selected townships to serve as primary sampling units, and stage three involved systematic random selection of two eligible respondents from each of the selected lins. During this process, 4412 men and women aged $\geq 60$ years were selected to serve as subjects, and among them, 4049 completed the initial interview in 1989.

In 1996, a second sample of 3041 subjects, 50-66 years, was drawn using the same process to extend the younger age range of the study cohort, and a total of 5131 subjects of the combined cohort completed the interview. The original or the combined cohort underwent a comprehensive in-home, face-to-face, structured questionnaire interview conducted by trained interviewers in 1989, 1993, 1996, 1999, 2003 and 2007. A flow chart of the TLSA has been presented previously $^{(13)}$. The 1999 survey, comprising nutritional assessment and the MNA items, was chosen as the baseline and the 2003 survey as the endpoint for the present study. The protocol of the surveys was reviewed and approved by government-appointed representatives. All participants gave informed consent, and the study was conducted according to the ethical standards set forth in the Declaration of Helsinki.

\section{Study subjects}

In the 1999 survey, 4440 of the 4915 subjects in the combined cohort completed the interview. Among them, 2890 subjects were aged $\geq 65$ years, but 150 who had incomplete MNA data were excluded. The remaining 2740 subjects served as the subjects in the present study. For cross-sectional regression analysis, the model included all the 2740 subjects. From longitudinal analysis, 250 subjects who had care need at baseline and seventy-six subjects who were lost to follow-up were excluded. The remaining 2414 subjects included 1964 who completed the 2003 survey and 450 who died before the 2003 survey. Among the 1964 subjects who completed the 2003 survey, 1728 had no care need and 236 had new care need. Among the 450 subjects who died before the 2003 survey, 421 were judged to have care need and twenty-nine to have no care need (Fig. 1). The judgements were based on the TLSA records, the mortality records of the study subjects maintained by the Health Promotion Administration, and the Household Registration data.

\section{Outcome measures}

The outcome measure in the present study was new onset of care need, which was defined as that in those who started needing formal care (care provided by trained carers in longterm care institutions or at homes) or informal care (care provided by family members or non-professional personnel)

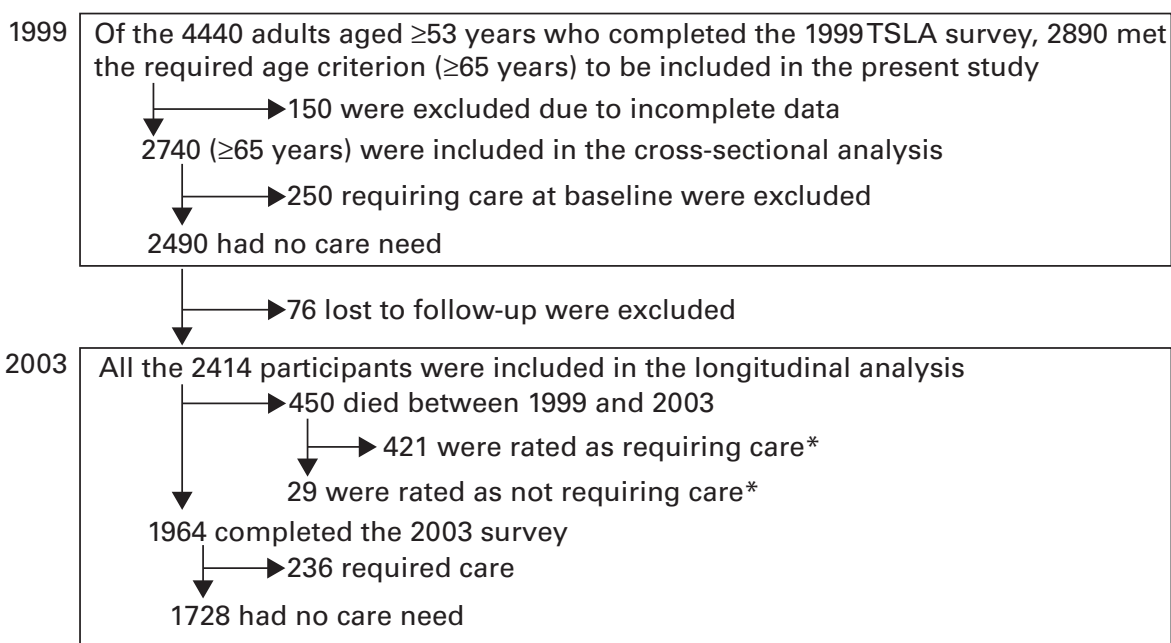

Fig. 1. Flow chart and care-need status of 2740 Taiwanese subjects aged $\geq 65$ years. * Based on Taiwan Longitudinal Study on Aging (TLSA) data or information provided by the Health Promotion Administration or the Household Registration records. 
or became Activities of Daily Living (ADL) dependent during the subsequent 4 years.

\section{Nutritional status assessment}

The nutritional status of each subject was rated with the LF and SF versions of the MNA-T1 and MNA-T2 at baseline. The MNA-T2 has been shown to perform slightly better than or at least equally to the MNA-T1 ${ }^{(6)}$. Data for all the items of the MNA, except fluid intake (1 point), were available in the datasets. Thus, the MNA scores in the present study were based on seventeen items for the MNA-T1 and sixteen items for the MNA-T2. However, the total scores were proportionately adjusted to the full 30 -point basis. A total score $\geq 24$ is considered as normal, $17-23.5$ as at risk of malnutrition, and $<17$ as malnourished. The MNA-SF, which was derived from a subset of the LF, has a score range of 0-14. A total score $\geq 12$ is considered as normal, $8-11$ as at risk of malnutrition, and $\leq 7$ as malnourished.

\section{Other variables}

All other variables were derived from the 1999 datasets. Education level was classified into three categories: $\leq 6$; $7-12 ; \geq 13$ years of formal education. BMI was calculated as weight divided by squared height in $\mathrm{kg} / \mathrm{m}^{2}$. Living arrangement was defined as living alone or cohabitation. Routine physical activity was classified into three categories: exercising 0 ; $\leq 2$; $\geq 3 \mathrm{~d} /$ week $(\geq 30 \mathrm{~min} / \mathrm{d}$ ). Functional status was based on ADL dependency; cognitive status was based on the education-adjusted Short Portable Mental Status Questionnaire scale; and depression status was rated with the 10-item Center for Epidemiologic Studies Depression (CES-D) scale. ADL reflect six daily self-care abilities including bathing, dressing/ undressing, self-feeding, functional transferring, ambulation and toileting ${ }^{(14)}$. An activity that one could not perform at all or had great difficulty in getting it done was considered dependent for that activity. A person with any domain of ADL dependency was considered as ADL dependent. The Short Portable Mental Status Questionnaire consists of ten items covering short-term and long-term memory, orientation, knowledge of current events and mathematical tasks, and the maximum score is $10^{(15)}$. In the present study, the score was education adjusted. Those who scored $<6,<7$ and $<8$ for $0,1-6$ and $>6$ years of formal education, respectively, were considered as cognitively impaired. The 10-item CES-D scale has been shown to preserve sensitivity and specificity as the 20-item original scale ${ }^{(16)}$ and to perform well in older Chinese ${ }^{(17)}$. The scale has a score range of $0-30$, and a score $\geq 10$ is considered as having depressive symptoms ${ }^{(18)}$.

\section{Statistical analyses}

All statistical analyses were carried out with the Statistical Package for the Social Sciences (SPSS version 19.0; SPSS, Inc.). Binary logistic regression analyses were carried out to determine the cross-sectional and longitudinal association of the MNA-T1 and MNA-T2 with care-need status controlled for age, sex, education level, living arrangement and routine physical activity. OR and 95\% CI were estimated for groups that were rated as at risk of malnutrition or as malnourished compared with those who were rated normal. Taking the MNA score as a continuous predictor, receiver operating characteristic curves and the AUC ${ }^{(19)}$ were generated to compare the ability of the four MNA (as at risk of nutrition + malnourished $v$. normal) to predict care need. A larger value of the AUC (the maximum value is 1.0) suggests a better predictive ability of the predictor. All statistical analyses were weighting adjusted according to study design (sampling ratio). Statistical significance for all analyses was accepted at $\alpha=0.05$.

\section{Results}

Table 1 summarises the characteristics of the subjects at baseline and endpoint. At baseline, the MNA-T1-LF rated fifty-seven (2.1\%) subjects as malnourished, $373(13.6 \%)$ as at risk of malnutrition and $2310(84.3 \%)$ as normal; $14.2 \%$ of the subjects had cognitive impairment and $30 \cdot 8 \%$ had depressive symptoms; at endpoint, the values were 17.0 and $28.0 \%$, respectively. At baseline, $9 \cdot 1 \%$ of the subjects had care need; at end point, $12.0 \%$ had care need.

Table 2 summarises the results of the univariate regression analysis of the association of care need with age, sex, education level, living arrangement, routine physical activity and nutritional status rated with the MNA at baseline and endpoint. Care need was strongly positively associated with age but negatively associated with education level. Poor nutrition greatly increased care need. The associations with sex and living arrangement were not significant.

Table 3 summarises the cross-sectional associations of nutritional status rated with the MNA with care need in older Taiwanese. Using the respective nutritionally normal group as the reference, the OR for needing care in those who were rated as at risk of malnutrition or malnourished were 2.50 and 14.2 for the MNA-T1-LF, 3.38 and 18.3 for the MNA-T2-LF, 2.02 and 7.23 for the MNA-T1-SF, and 2.61 and 8.84 for the MNA-T2-SF (all $P<0.001$ ), controlled for age, sex, education level, living arrangement and routine physical activity; the $-2 \log$ likelihood values were 999.8, 989.7, $1010 \cdot 8$ and $1003 \cdot 2$ and the Nagelkerke $R^{2}$ values were $0 \cdot 225$, $0 \cdot 235,0 \cdot 215$ and $0 \cdot 222$, respectively.

Table 4 summarises the longitudinal associations of baseline nutritional status rated with the LF and SF versions of the MNA-T1 and MNA-T2 with care need 4 years later in older Taiwanese. Using the respective nutritionally normal group (at baseline) as the reference, the OR for needing care in those who were rated as at risk of malnutrition or malnourished were 2.04 and 3.33 for the MNA-T1-LF, 2.10 and 5.35 for the MNA-T2-LF, 1.49 and 2.48 for the MNA-T1-SF, and 1.80 and 3.44 for the MNA-T2-SF (all $P<0.05$ ), controlled for the same variables as in the cross-sectional model; the -2 $\log$ likelihood values were 1873.5, 1872.1, $1882 \cdot 0$ and $1871 \cdot 2$ and the Nagelkerke $R^{2}$ values were $0 \cdot 190,0 \cdot 191,0 \cdot 184$ and $0 \cdot 192$, respectively. 
Table 1. Characteristics of the 2740 Taiwanese subjects at baseline ( $\geq 65$ years old) and at endpoint

(Number of subjects and percentages, mean values and standard deviations; $n$ 1964)

\begin{tabular}{|c|c|c|c|c|}
\hline \multirow[b]{2}{*}{ Variables } & \multicolumn{2}{|c|}{ At baseline } & \multicolumn{2}{|c|}{ At endpoint } \\
\hline & $n$ & $\%$ & $n$ & $\%$ \\
\hline \multicolumn{5}{|l|}{$\operatorname{Sex}(n)$} \\
\hline Men & 1516 & $55 \cdot 3$ & 1054 & $53 \cdot 7$ \\
\hline Women & 1224 & $44 \cdot 7$ & 910 & $46 \cdot 3$ \\
\hline \multicolumn{5}{|l|}{ Age (years) ${ }^{\star}$} \\
\hline $65-74$ & 1556 & $56 \cdot 8$ & 1275 & 64.9 \\
\hline$\geq 75$ & 1184 & $43 \cdot 2$ & 689 & $35 \cdot 1$ \\
\hline \multicolumn{5}{|c|}{ Formal education (years) } \\
\hline$\leq 6$ & 2141 & $78 \cdot 1$ & 1512 & $77 \cdot 0$ \\
\hline $7-12$ & 442 & $16 \cdot 1$ & 338 & $17 \cdot 2$ \\
\hline$\geq 13$ & 157 & $5 \cdot 7$ & 114 & 5.8 \\
\hline \multicolumn{5}{|l|}{ Smoking status } \\
\hline Never smoke & 1545 & $56 \cdot 4$ & 1127 & $57 \cdot 4$ \\
\hline Past smoker & 569 & $20 \cdot 8$ & 500 & $25 \cdot 5$ \\
\hline Current smoker & 626 & $22 \cdot 8$ & 337 & $17 \cdot 2$ \\
\hline \multicolumn{5}{|l|}{ Alcohol drinking } \\
\hline Do not drink & 2140 & $78 \cdot 1$ & 1553 & $79 \cdot 1$ \\
\hline$<1$ time/week & 245 & 8.9 & 194 & 9.9 \\
\hline$\geq 1$ time/week & 355 & $13 \cdot 0$ & 217 & 11.0 \\
\hline \multicolumn{5}{|c|}{ Betel quid chewing habit } \\
\hline Never chew & 2502 & $91 \cdot 3$ & 1807 & $92 \cdot 0$ \\
\hline Past chewer & 119 & $4 \cdot 3$ & 96 & 4.9 \\
\hline Current chewer & 119 & 4.3 & 61 & 3.1 \\
\hline \multicolumn{5}{|c|}{ Routine physical activity } \\
\hline Not active & 1064 & $38 \cdot 8$ & 693 & $35 \cdot 3$ \\
\hline$\leq 2$ times/week & 156 & $5 \cdot 7$ & 87 & 4.4 \\
\hline$\geq 3$ times/week & 1520 & 55.5 & 1184 & $60 \cdot 3$ \\
\hline \multicolumn{5}{|l|}{ Cohabitation } \\
\hline Yes & 2436 & 88.9 & 1743 & $88 \cdot 7$ \\
\hline No & 304 & $11 \cdot 1$ & 221 & $11 \cdot 3$ \\
\hline \multicolumn{5}{|c|}{ Nutritional statust (MNA-T1-LF) } \\
\hline$\leq 16 \cdot 5$ & 57 & $2 \cdot 1$ & 11 & 0.6 \\
\hline $17-23.5$ & 373 & $13 \cdot 6$ & 190 & 9.7 \\
\hline$\geq 24$ & 2310 & $84 \cdot 3$ & 1763 & 89.8 \\
\hline Mean & \multicolumn{2}{|c|}{$26 \cdot 3$} & \multicolumn{2}{|c|}{26.9} \\
\hline SD & \multicolumn{2}{|c|}{$3 \cdot 1$} & \multicolumn{2}{|c|}{2.5} \\
\hline \multicolumn{5}{|c|}{ Cognitive status (SPMSQ score) $\ddagger$} \\
\hline Normal & 2351 & $85 \cdot 8$ & 1630 & $83 \cdot 0$ \\
\hline Impaired & 389 & $14 \cdot 2$ & 334 & $17 \cdot 0$ \\
\hline Mean score & \multicolumn{2}{|c|}{8.3} & \multicolumn{2}{|c|}{7.9} \\
\hline SD & & & & \\
\hline CES-D score & & & & \\
\hline$<10$ & 1896 & $69 \cdot 2$ & 1415 & $72 \cdot 0$ \\
\hline$\geq 10$ & 844 & $30 \cdot 8$ & 549 & $28 \cdot 0$ \\
\hline Mean score & & & & \\
\hline SD & & & & \\
\hline No. of ADL & & & & \\
\hline 0 & 2525 & $92 \cdot 2$ & 1772 & $90 \cdot 2$ \\
\hline $1-3$ & 100 & 3.6 & 88 & 4.5 \\
\hline $4-6$ & 115 & 4.2 & 104 & $5 \cdot 3$ \\
\hline Mean no. of ADL & & & & \\
\hline SD & & & & \\
\hline Need care§ & & & & \\
\hline No & 2490 & $90 \cdot 9$ & 1728 & 88.0 \\
\hline Yes & 250 & $9 \cdot 1$ & 236 & $12 \cdot 0$ \\
\hline
\end{tabular}

MNA-T1-LF, Mini Nutritional Assessment-Taiwan version 1-long form; SPMSQ, Short Portable Mental Status Questionnaire; CES-D, Center for Epidemiologic Studies Depression; ADL, Activities of Daily Living.

* 65 and 75 years of age correspond to birth years 1934 and 1924, respectively.

†All values were those of nutritional status rated with the MNA-T1-LF at baseline. The distributions of nutritional status rated with other MNA versions are given in Supplementary Appendix A (available online).

$\ddagger$ Based on education-adjusted SPMSQ score: subjects who scored $<6,<7$ and $<8$ for $0,1-6$ and $>6$ years of formal education, respectively, were considered as cognitively impaired.

SIncluding those who needed formal care (care provided by trained carers) or informal care (care provided by non-professional persons including family members) or were ADL dependent.
Fig. 2 shows the receiver operating characteristic curves generated for each of the MNA against care need at baseline. The AUC was $0.762(95 \%$ CI $0.730,0.794)$ for the MNA-T1-LF, 0.771 (95\% CI 0.739, 0.802) for the MNA-T2-LF, 0.720 (95\% CI $0.685,0.755)$ for the MNA-T1-SF, and 0.732 (95\% CI 0.697 , $0 \cdot 767$ ) for the MNA-T2-SF (all $P<0.001$ ). The scoring patterns and the nutritional status rated with the MNA versions are given in Supplementary Appendix A (available online).

\section{Discussion}

The results of the present study suggest that the MNA (both forms and both versions) can effectively predict concurrent and subsequent care need in older people. Those who were rated as at risk of malnutrition were found to have greater current need for care and have increased need for care during the subsequent 4 years. These results suggest that the MNA, whether LF or SF, might have a greater clinical value than previously recognised.

\section{Cross-sectional and longitudinal associations}

MNA scores usually reflect frailty in older people. Malnutrition rated with the MNA is associated with functional dependency, cognitive impairment, depression and difficulties in chewing and swallowing ${ }^{(9)}$. Older patients from the general practice found to be at risk of malnutrition rated with the MNA had higher need for meals-on-wheels and home care for shopping than those who were well nourished ${ }^{(20)}$. Cereda et $a l^{(10)}$ observed that the MNA was a good predictor of functional status in institutionalised older people at risk of malnutrition.

The MNA can also predict subsequent functional decline. Older patients from the general practice rated as at risk of malnutrition with the MNA at baseline had a higher tendency for non-participation and a higher prevalence of hospitalisation 6 months later ${ }^{(20)}$. The MNA-SF was also found to be able to predict functional decline (defined as a loss of $10 \%$ of the Barthel index score during 2-week hospitalisation) in patients admitted to acute care ${ }^{(21)}$. Those who were rated as at risk of malnutrition had worse clinical features, longer length of hospital stay and considerable functional decline. Studies carried out by our group have shown that both the MNA-LF and MNA-SF were associated with greater ADL or Instrumental ADL dependency at baseline and were able to predict functional decline (measured as increasing number of ADL or Instrumental ADL dependency) 4 years later in older Taiwanese. The longitudinal associations existed even among older people who were free of ADL or Instrumental ADL dependency at baseline ${ }^{(22)}$. The MNA-T2 performed better than or at least equally to the $\mathrm{MNA}-\mathrm{T} 1^{(23)}$ in predicting the decline. We also observed that the ADL complemented the MNA or the MNA-SF in predicting follow-up mortality risk in older people ${ }^{(24)}$. These results suggest that the MNA is able to predict $\mathrm{ADL}$ or Instrumental ADL dependency, in addition to rating current nutritional status in older people. Poor nutrition rated with the MNA has also been observed to be associated with poorer concurrent cognitive status and higher subsequent cognitive decline in older Taiwanese ${ }^{(25)}$. 
Table 2. Results of the univariate regression analysis of the associations of the independent variables with care need of a sample of Taiwanese subjects ( $n 2740$, aged $\geq 65$ years at baseline) and at endpoint ( $n$ 2414) (weighted) ( $\beta$ Coefficients, odds ratios and $95 \%$ confidence intervals)

\begin{tabular}{|c|c|c|c|c|c|c|c|c|}
\hline \multirow[b]{2}{*}{ Variables } & \multicolumn{4}{|c|}{ Care need at baseline (1999) } & \multicolumn{4}{|c|}{ Care need at endpoint (2003) } \\
\hline & $\beta$ & OR & $95 \% \mathrm{Cl}$ & $P$ & $\beta$ & OR & $95 \% \mathrm{Cl}$ & $P$ \\
\hline Age & 0.116 & $1 \cdot 12$ & $1 \cdot 10,1 \cdot 15$ & $<0.001$ & 0.134 & $1 \cdot 14$ & $1 \cdot 12,1 \cdot 17$ & $<0.001$ \\
\hline Sex & 0.204 & 1.23 & $0.90,1.67$ & 0.196 & -0.007 & 0.99 & $0.81,1.23$ & 0.948 \\
\hline Education level & -0.053 & 0.95 & $0.91,0.99$ & 0.006 & -0.047 & 0.95 & $0.93,0.98$ & $<0.001$ \\
\hline Living alone & 0.416 & 1.52 & $0.95,2.36$ & 0.065 & 0.264 & $1 \cdot 30$ & $0.94,1.81$ & 0.118 \\
\hline \multicolumn{9}{|l|}{ Physical activity } \\
\hline$\geq 3$ times/week & & 1.00 & & & & 1.00 & & \\
\hline$\leq 2$ times/week & 0.428 & 1.54 & $0.69,3.42$ & 0.296 & 0.217 & 1.24 & $0.79,1.96$ & 0.350 \\
\hline Not active & 1.478 & $4 \cdot 38$ & $3 \cdot 09,6 \cdot 23$ & $<0.001$ & 0.683 & 1.98 & $1.59,2.46$ & $<0.001$ \\
\hline \multicolumn{9}{|l|}{ Nutritional status* } \\
\hline Normal & & 1.00 & & & & 1.00 & & \\
\hline At risk & 1.410 & $4 \cdot 10$ & $2 \cdot 85,5 \cdot 89$ & $<0.001$ & 0.982 & $2 \cdot 67$ & $1.99,3.60$ & $<0.001$ \\
\hline Malnourished & $3 \cdot 183$ & $24 \cdot 1$ & $12 \cdot 5,46 \cdot 5$ & $<0.001$ & $1 \cdot 441$ & $4 \cdot 22$ & $1.61,11.07$ & 0.003 \\
\hline
\end{tabular}

* Rated with the Mini Nutritional Assessment-Taiwan version 1.

\section{Prediction of care need or higher level of care}

Severe functional decline and cognitive impairment can lead to frailty and increasing dependency. In addition to being a good predictor of functional status in institutionalised older people at risk of malnutrition, the MNA was found to be reliable for identifying the at-risk institutionalised older people who needed higher-level care ${ }^{(10)}$. The MNA-SF was among a list of scales that defined frailty and the need for care among community-dwelling older people in Sweden ${ }^{(26)}$. Dent et $a{ }^{(27)}$ have recently concluded in a review that nutritional status rated with nutrition screening tools, particularly the MNA-LF and MNA-SF, is associated with subsequent functional decline and need for higher-level care in older people. The present study has further shown that both the MNA-LF and MNA-SF of both T1 and T2 versions are effective at rating current care status and at predicting care need during the subsequent 4 years in older Taiwanese. Thus, in both Eastern and Western populations, the MNA-LF and MNA-SF have been shown to rate current functional and cognitive status and to predict subsequent functional and cognitive declines, the major determinants of care need, institutionalisation or need for higher-level care in older people $\mathrm{e}^{(28-30)}$. These studies suggest that the MNA is a tool that not only rates malnutrition risk, but can also predict subsequent functional decline, cognitive impairment and the need for care or higher-level care.

\section{The short-form Mini Nutritional Assessment has advantages}

Although the MNA-LF can predict subsequent care need with greater accuracy than the MNA-SF, the MNA-SF would still be a preferred tool in both clinical and community settings simply because it is much easier and time saving $(5 v, 20-30 \mathrm{~min})$ to use. The MNA-SF has much fewer items (6v.18) and it does not include the difficult and time-consuming items such as fluid and protein intakes. It appears worthwhile to trade a bit of accuracy for a large gain on usability. Simplicity and easy execution are important features of successful screening tools in both clinical and community settings.
The present results also reveal that the T2 versions performed slightly better than the T1 versions. Although the improvement in performance is only moderate, the improvement in utility is significant. The MNA-T2-SF substituted CC for BMI. Measuring CC is much easier than measuring weight and height, especially in people who have mobility limitations or disability. CC data could also be more accurate than BMI data in older people. Thus, the MNA-T2-SF is favoured over the MNA-T1-SF.

\section{Implications}

We have demonstrated with a large population-representative sample that the MNA not only can predict current care need but can also predict subsequent care need in older people. These results suggest that the MNA has the properties to serve as a tool for screening care need in older people. Given that most populations in the world are ageing rapidly, such tools are urgently needed. Of course, further improvements are needed before these tools can become acceptable care need-predicting scales. For example, it does not include socio-economic, cultural or healthcare system factors. However, the MNA-SF does possess the basic or major features for being used as a care need-predicting tool.

\section{Limitations of the study}

The present study has some limitations. (a) A major limitation is that interview surveys in the TLSA took place every 4 years. Therefore, the time of onset of care need could not be accurately pinpointed and analysed for its association with the MNA scores in the present study. (b) Attrition is an inherent issue in longitudinal studies involving older people. Whether these subjects are included in the statistical analyses can have a great impact on results. In the present study, we included those who died but not those (seventy-six subjects) who were lost to follow-up in the longitudinal analysis. For those who died, we used the care arrangement at the time of death recorded in the datasets or mortality records to 
Table 3. Cross-sectional associations of nutritional status rated with the Mini Nutritional Assessment (MNA) or the short-form MNA with care need in 2740 Taiwanese subjects aged $\geq 65$ years at baseline (weighted)

( $\beta$ Coefficients, odds ratios and $95 \%$ confidence intervals)

\begin{tabular}{|c|c|c|c|c|c|c|c|c|c|c|c|c|c|c|c|c|}
\hline \multirow[b]{2}{*}{$\underline{\text { Variables }}$} & \multicolumn{4}{|c|}{ Model 1: MNA-T1-LF } & \multicolumn{4}{|c|}{ Model 2: MNA-T2-LF } & \multicolumn{4}{|c|}{ Model 3: MNA-T1-SF } & \multicolumn{4}{|c|}{ Model 4: MNA-T2-SF } \\
\hline & $\beta$ & OR & $95 \% \mathrm{Cl}$ & $P$ & $\beta$ & OR & $95 \% \mathrm{Cl}$ & $P$ & $\beta$ & OR & $95 \% \mathrm{Cl}$ & $P$ & $\beta$ & OR & $95 \% \mathrm{Cl}$ & $P$ \\
\hline Age $^{*}$ & 0.096 & $1 \cdot 10$ & $1.07,1.13$ & $<0.001$ & 0.099 & $1 \cdot 10$ & $1.08,1 \cdot 13$ & $<0.001$ & 0.095 & $1 \cdot 10$ & $1.07,1.13$ & $<0.001$ & 0.098 & $1 \cdot 10$ & $1.08,1.13$ & $<0.001$ \\
\hline Sext & 0.123 & 1.13 & $0.78,1.64$ & 0.517 & 0.131 & 1.14 & $0.78,1.66$ & 0.493 & 0.106 & $1 \cdot 11$ & $0.77,1.61$ & 0.576 & 0.131 & 1.14 & $0.79,1.65$ & 0.491 \\
\hline Education level ${ }^{*}$ & 0.022 & 1.02 & $0.98,1.07$ & 0.339 & 0.025 & 1.03 & $0.98,1.07$ & 0.271 & 0.017 & 1.02 & $0.97,1.06$ & 0.457 & 0.018 & 1.02 & $0.97,1.06$ & 0.412 \\
\hline Living alone† & 0.391 & 1.48 & $0.91,2.39$ & 0.111 & 0.354 & 1.42 & $0.88,2.31$ & 0.153 & 0.395 & 1.48 & $0.92,2.40$ & 0.107 & 0.367 & 1.44 & $0.89,2.34$ & 0.135 \\
\hline \multicolumn{17}{|l|}{ Physical activity } \\
\hline$\geq 3$ times/week & & 1.00 & & & & 1.00 & & & & 1.00 & & & & 1.00 & & \\
\hline$\leq 2$ times/week & 0.367 & 1.44 & $0.63,3.32$ & 0.387 & 0.322 & 1.38 & $0.60,3.20$ & 0.452 & 0.353 & 1.42 & $0 \cdot 61,3.30$ & 0.410 & 0.356 & 1.43 & $0.62,3.30$ & 0.405 \\
\hline Not active & 1.079 & 2.94 & $2 \cdot 00,4.32$ & $<0.001$ & 1.013 & 2.76 & $1.87,4.06$ & $<0.001$ & 1.150 & $3 \cdot 16$ & $2 \cdot 16,4 \cdot 61$ & $<0.001$ & 1.111 & 3.04 & $2.08,4.44$ & $<0.001$ \\
\hline \multicolumn{17}{|c|}{ Nutritional status rated with the MNA } \\
\hline Normal & & 1.00 & & & & 1.00 & & & & 1.00 & & & & 1.00 & & \\
\hline At risk & 0.916 & 2.50 & $1.69,3.70$ & $<0.001$ & 1.219 & 3.38 & $2.30,4.99$ & $<0.001$ & 0.704 & 2.02 & $1.39,2.95$ & $<0.001$ & 0.960 & $2 \cdot 61$ & $1 \cdot 81,3.77$ & $<0.001$ \\
\hline Malnourished & 2.655 & $14 \cdot 23$ & $6 \cdot 94,29 \cdot 16$ & $<0.001$ & 2.905 & 18.26 & $7.93,42.02$ & $<0.001$ & 1.978 & $7 \cdot 23$ & $4 \cdot 21,12 \cdot 41$ & $<0.001$ & $2 \cdot 180$ & 8.84 & $4 \cdot 79,16 \cdot 32$ & $<0.001$ \\
\hline$-2 \mathrm{LL}$ & & 999.8 & & & & 989.7 & & & & $1010 \cdot 8$ & & & & 1003.2 & & \\
\hline Nagelkerke $R^{2}$ & & 0.225 & & & & 0.235 & & & & 0.215 & & & & 0.222 & & \\
\hline
\end{tabular}

MNA-T1-LF, MNA-Taiwan version 1-long form; MNA-T2-LF, MNA-Taiwan version 2-long form; MNA-T1-SF, MNA-Taiwan version 1-short form; MNA-T2-SF, MNA-Taiwan version 2-short form; -2LL, -2 log likelihood.

† A Age and education level were continuous variables.

Table 4. Longitudinal associations of the baseline Mini Nutritional Assessment (MNA) status with new care need 4 years later in 2414 Taiwanese subjects aged $\geq 65$ years (weighted) ( $\beta$ Coefficients, odds ratios and $95 \%$ confidence intervals)

\begin{tabular}{|c|c|c|c|c|c|c|c|c|c|c|c|c|c|c|c|c|}
\hline \multirow[b]{2}{*}{ Variables } & \multicolumn{4}{|c|}{ Model 1: MNA-T1-LF } & \multicolumn{4}{|c|}{ Model 2: MNA-T2-LF } & \multicolumn{4}{|c|}{ Model 3: MNA-T1-SF } & \multicolumn{4}{|c|}{ Model 4: MNA-T2-SF } \\
\hline & $\beta$ & OR & $95 \% \mathrm{Cl}$ & $P$ & $\beta$ & OR & $95 \% \mathrm{Cl}$ & $P$ & $\beta$ & OR & $95 \% \mathrm{Cl}$ & $P$ & $\beta$ & OR & $95 \% \mathrm{Cl}$ & $P$ \\
\hline Age $^{*}$ & 0.129 & $1 \cdot 14$ & $1 \cdot 12,1 \cdot 16$ & $<0.001$ & 0.130 & $1 \cdot 14$ & $1 \cdot 12,1 \cdot 16$ & $<0.001$ & 0.129 & $1 \cdot 14$ & $1 \cdot 12,1 \cdot 16$ & $<0.001$ & 0.130 & $1 \cdot 14$ & $1 \cdot 12,1 \cdot 16$ & $<0.001$ \\
\hline Sext & -0.149 & 0.86 & $0.67,1.11$ & 0.246 & -0.138 & 0.87 & $0.68,1.12$ & 0.283 & -0.156 & 0.86 & $0.67,1.10$ & 0.221 & -0.137 & 0.87 & $0.68,1.12$ & 0.286 \\
\hline Education level ${ }^{*}$ & -0.009 & 0.99 & $0.96,1.02$ & 0.570 & -0.008 & 0.99 & $0.96,1.02$ & 0.610 & -0.012 & 0.99 & $0.96,1.02$ & 0.421 & -0.011 & 0.99 & $0.96,1.02$ & 0.442 \\
\hline Living alone† & 0.149 & $1 \cdot 16$ & $0.81,1.67$ & 0.418 & 0.133 & $1 \cdot 14$ & $0.80,1.64$ & 0.471 & 0.165 & $1 \cdot 18$ & $0.82,1.69$ & 0.368 & 0.148 & $1 \cdot 16$ & $0.81,1.67$ & 0.422 \\
\hline \multicolumn{17}{|l|}{ Physical activity } \\
\hline$\geq 3$ times/week & & 1.00 & & & & 1.00 & & & & 1.00 & & & & 1.00 & & \\
\hline$\leq 2$ times/week & 0.218 & 1.24 & $0.77,2.02$ & 0.378 & 0.211 & 1.23 & $0.76,2.01$ & 0.395 & 0.227 & 1.25 & $0.77,2.03$ & 0.358 & 0.224 & 1.25 & $0.77,2.03$ & 0.366 \\
\hline Not active & 0.550 & 1.73 & $1 \cdot 36,2 \cdot 21$ & $<0.001$ & 0.538 & 1.71 & $1 \cdot 34,2 \cdot 18$ & $<0.001$ & 0.582 & 1.79 & $1 \cdot 41,2 \cdot 28$ & $<0.001$ & 0.557 & 1.74 & $1 \cdot 37,2 \cdot 22$ & $<0.001$ \\
\hline \multicolumn{17}{|c|}{ Nutritional status rated with the MNA } \\
\hline Normal & & 1.00 & & & & 1.00 & & & & 1.00 & & & & 1.00 & & \\
\hline At risk & 0.714 & 2.04 & $1 \cdot 48,2 \cdot 83$ & $<0.001$ & 0.742 & $2 \cdot 10$ & $1.50,2.95$ & $<0.001$ & 0.396 & 1.49 & $1 \cdot 12,1.97$ & 0.006 & 0.587 & 1.80 & $1 \cdot 36,2 \cdot 38$ & $<0.001$ \\
\hline Malnourished & 1.204 & 3.33 & $1.20,9.25$ & 0.021 & 1.677 & $5 \cdot 35$ & $1.46,19.61$ & 0.011 & 0.907 & 2.48 & $1.31,4.68$ & 0.005 & 1.236 & 3.44 & $1.63,7.28$ & 0.001 \\
\hline$-2 \mathrm{LL}$ & & $1873 \cdot 5$ & & & & $1872 \cdot 1$ & & & & $1882 \cdot 0$ & & & & $1871 \cdot 2$ & & \\
\hline Nagelkerke $R^{2}$ & & 0.190 & & & & 0.191 & & & & 0.184 & & & & 0.192 & & \\
\hline
\end{tabular}

MNA-T1-LF, MNA-Taiwan version 1-long form; MNA-T2-LF, MNA-Taiwan version 2-long form; MNA-T1-SF, MNA-Taiwan version 1-short form; MNA-T2-SF, MNA-Taiwan version 2-short form; -2LL, -2 log likelihood. *Age and education level were continuous variables.

† Male was used as reference for sex and cohabitation was used as reference for living arrangement. 


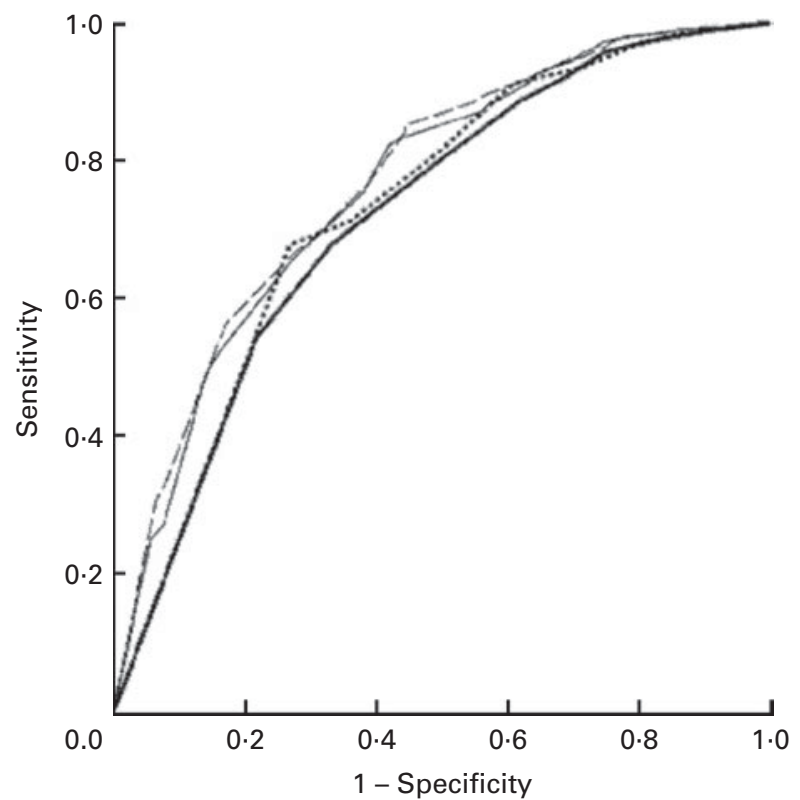

Fig. 2. Receiver operating characteristic curves generated for the Mini Nutritional Assessment-Taiwan version 1-long form (MNA-T1-LF; - ), Mini Nutritional Assessment-Taiwan version 2-long form (MNA-T2-LF; --), Mini Nutritional Assessment-Taiwan version 1-short form (MNA-T1-SF; - ) and Mini Nutritional Assessment-Taiwan version 2-short form (MNA-T2-SF; --.-), respectively, against care need at baseline in 2740 Taiwanese subjects aged $\geq 65$ years. The AUC was $0.762(95 \% \mathrm{Cl} 0.730,0.794)$ for the MNA-T1-LF, 0.771 (95\% Cl 0.739, 0.802) for the MNA-T2-LF, 0.720 (95\% Cl 0.685, $0.755)$ for the MNA-T1-SF, and $0.732(95 \% \mathrm{Cl} 0.697,0.767)$ for the MNAT2-SF (all $P<0.001$ ).

judge their probable care need. However, there was no such information for those who were lost to follow-up. (c) Most data were self-reported. Self-reports generally have good accuracy, but errors due to incorrect recall of information or unwillingness to reveal personal information are unavoidable. (d) Functional condition and cognitive status were evaluated with scales not clinically diagnosed. Although the scales are well established, individual differences in answering the questions included in the scales are unavoidable. (e) Care need or care arrangement is influenced by many conditions. Culture, socio-economic factors and personal view/ideology may influence the onset of care need or care arrangement. These potential confounders could not be totally controlled. (f) Fluid intake (1 point) was not assessed in the surveys. However, the TLSA sample was drawn from the general population, not from institutionalised or frail older population, and we also proportionately adjusted to the full 30-point basis. The overall impact on study results should be minimal.

\section{Conclusion}

The MNA-LF and MNA-SF of both T1 and T2 versions, in addition to rating the risk of malnutrition, can also rate functional and cognitive status, predict functional and cognitive impairments, and predict subsequent care need in older people. Among the four MNA, the MNA-T2-SF appears to be most promising because of its performance, simplicity and efficiency. The MNA-T2-SF may have the basic features for being developed into a more complete tool for predicting care need, a tool urgently needed in this rapidly ageing world. Early detection is important to allow targeted interventions and should be a key factor in geriatric assessment.

\section{Supplementary material}

To view supplementary material for this article, please visit http://dx.doi.org/10.1017/S0007114514000142

\section{Acknowledgements}

The present study was based on data from the TLSA provided by the Health Promotion Administration (HPA), Department of Health, Taiwan. Descriptions or conclusions reported herein do not represent the viewpoint of the HPA.

The study did not receive financial support from any source.

The authors' contributions are as follows: A. C. T. conceived the study design and drafted the manuscript; W.-C. H. carried out the statistical analyses; J.-Y. W. helped with the statistical analyses and interpretation of the data. All authors reviewed and approved the manuscript.

None of the authors has any conflicts of interest to declare.

\section{References}

1. Guigoz Y, Vellas BJ \& Garry PJ (1994) The Mini Nutritional Assessment (MNA): a practical assessment tool for grading the nutritional state of elderly patients. In Facts and Research in Gerontology, Nutrition Supplement, pp. 15-60 [BJ Vellas, Y Guigoz, PJ Garry and JL Albarede, editors]. New York: Serdi Publishing Company.

2. Guigoz Y, Lauque S \& Villas BJ (2002) Identifying the elderly at risk for malnutrition. The Mini Nutritional Assessment. Clin Geriatr Med 18, 737-757.

3. Vellas B, Villars H, Abellan G, et al. (2006) Overview of the MNA - its history and challenge. J Nutr Health Aging 10, 456-463.

4. Rubenstein LZ, Harker JO, Salva A, et al. (2001) Screening for undernutrition in geriatric practice: developing the shortform mini-nutritional assessment (MNA-SF). J Gerontol $A$ Biol Sci Med Sci 56, M366-M372.

5. Kaiser MJ, Bauer JM, Ramsch C, et al. (2009) Validation of the Mini Nutritional Assessment short-form (MNA-SF): a practical tool for identification of nutritional status. $J$ Nutr Health Aging 13, 782-788.

6. Tsai AC, Chang TL, Wang YC, et al. (2010) Populationspecific short-form mini nutritional assessment with body mass index or calf circumference can predict risk of malnutrition in community-living or institutionalized elderly in Taiwan. J Am Dietet Assoc 110, 1328-1334.

7. Tsai AC, Ho CS \& Chang MC (2007) Population-specific anthropometric cut-points improve the functionality of the Mini Nutritional Assessment (MNA) in elderly Taiwanese. Asia Pac J Clin Nutr 16, 656-662.

8. Tsai AC, Ho CS \& Chang MC (2008) Assessing the prevalence of malnutrition with the Mini Nutritional Assessment (MNA) in a nationally representative sample of elderly Taiwanese. J Nutr Health Aging 12, 239-243.

9. Guigoz Y (2006) The Mini Nutritional Assessment (MNA) review of the literature - what does it tell us? J Nutr Health Aging 10, 466-487. 
10. Cereda E, Valzolgher L \& Pedrolli C (2008) Mini nutritional assessment is a good predictor of functional status in institutionalized elderly at risk of malnutrition. Clin Nutr 27, 700-705.

11. Tsai AC, Chang TL \& Wang JY (2013) Short-form MiniNutritional Assessment with either BMI or calf circumference is effective in rating the nutritional status of elderly Taiwanese - results of a national cohort study. Br J Nutr 110, 1126-1132.

12. Bureau of Health Promotion \& Department of Health, Taiwan (1989) Survey of the elderly in Taiwan. http:// www.bhp.doh.gov.tw/BHPnet/Portal/Them.aspx?No $\frac{1}{4} 200712$ 270002 (accessed 30 June 2013).

13. Tsai AC \& Chang TL (2011) The effectiveness of BMI, calf circumference and mid-arm circumference in predicting subsequent mortality risk in elderly Taiwanese. Br J Nutr 105, 275-281.

14. Katz S, Ford AB, Moskowitz RW, et al. (1963) Studies of illness in the aged. The index of ADLs: a standardized measure of biological and psychosocial function. JAMA 185, 914-919.

15. Pfeiffer E (1975) A short portable mental status questionnaire for the assessment of organic brain deficit in elderly patients. J Am Geriatr Soc 23, 433-441.

16. Kohout FJ, Berkman LF, Evans DA, et al. (1993) Two shorter forms of the CES-D (Center for Epidemiological Studies Depression) depression symptoms index. J Aging Health 5, 179-193.

17. Boey KW (1999) Cross-validation of a short form of the CES-D in Chinese elderly. Int J Geriatr Psychiatry 14, 608-617.

18. Andresen EM, Malmgren JA, Carter WB, et al. (1994) Screening for depression in well older adults: evaluation of a short form of the CES-D (Center for Epidemiologic Studies Depression Scale). Am J Prev Med 10, 77-84.

19. Zweig MH \& Campbell G (1993) Receiver-operating characteristic (ROC) plots: a fundamental evaluation tool in clinical medicine. Clin Chem 39, 561-577.

20. Beck AM, Ovesen L \& Schroll M (2001) A six months prospective follow-up of $65+-y-o l d$ patients from general practice classified according to nutritional risk by the Mini Nutritional Assessment. Eur J Clin Nutr 55, 1028-1033.

21. Salvi F, Giorgi R, Grilli A, et al. (2008) Mini Nutritional Assessment (short form) and functional decline in older patients admitted to an acute medical ward. Aging Clin Exp Res 20, 322-328.

22. Lee LC \& Tsai AC (2012) Mini-Nutritional Assessment predicts functional decline of elderly Taiwanese: result of a population-representative sample. Br J Nutr 107, $1707-1713$

23. Lee LC \& Tsai AC (2012) Mini-Nutritional-Assessment (MNA) without body mass index (BMI) predicts functional disability in elderly Taiwanese. Arch Geront Geriatr 54, e405-e410.

24. Tsai AC, Lee LC \& Wang JY (2013) Complementarity of the Mini-Nutritional Assessment and activities of daily living for predicting follow-up mortality risk in elderly Taiwanese. Br J Nutr 109, 658-666.

25. Huang CJ \& Tsai AC (2013) The impact of nutritional status on cognitive decline in elderly Taiwanese. Taiwan J Pub Health 32, 42-51.

26. Bravell ME, Westerlind B, Midlov P, et al. (2011) How to assess frailty and the need for care? Report from the Study of Health and Drugs in the Elderly (SHADES) in community dwellings in Sweden. Arch Geront Geriatr 53, 40-45.

27. Dent E, Visvanathan R, Piantadosi C, et al. (2012) Nutritional screening tools as predictors of mortality, functional decline, and move to higher level care in older people: a systematic review. J Nut Geront Geriat 31, 97-145.

28. Tsai AC \& Lai TM (2011) Predicting the need of formal care in Taiwan: analysis of a national random sample. Arch Geront Geriatr 53, 298-302.

29. Gaugler JE, Duval S, Anderson KA, et al. (2007) Predicting nursing home admission in the US: a meta-analysis. BMC Geriatr 7, 13.

30. de Saint-Hubert M, Schoevaerdts D, Cornette P, et al. (2010) Predicting functional adverse outcomes in hospitalized older patients: a systematic review of screening tools. J Nutr Health Aging 14, 394-399. 